\title{
Domain decomposition methods for nonlinear transmission conditions
}

\author{
Matthias A. F. Gsell, Olaf Steinbach \\ Institut für Numerische Mathematik, TU Graz, \\ Steyrergasse 30, 8010 Graz, Austria
}

\begin{abstract}
We consider the transformation of quasilinear partial differential equations to a coupled system of linear equations, but with nonlinear transmission conditions on the interfaces. After deriving a variational formulation, we will discuss Mortar finite element discretization strategies and present a numerical example.
\end{abstract}

\section{Model problem}

As a model problem we consider the following quasilinear boundary value problem in a bounded domain $\Omega \subset \mathbb{R}^{d}(d=2,3)$ with Lipschitz boundary $\Gamma=\partial \Omega=\bar{\Gamma}_{D} \cup \bar{\Gamma}_{N}$ and $\Gamma_{D} \cap \Gamma_{N}=\emptyset$ to find $p$ such that

$$
\left.\begin{array}{rlrl}
-\nabla \cdot\left(k_{r}(\theta(p)) \nabla p\right) & =f & & \text { in } \Omega, \\
p & =0 & & \text { on } \Gamma_{D}, \\
k_{r}(\theta(p)) \nabla p \cdot \mathbf{n} & =g_{N} & & \text { on } \Gamma_{N} \cdot
\end{array}\right\}
$$

This model problem can be seen as a simplification of the stationary Richards equation without gravity, see [1].

If $k_{r}$ only depends on $\theta$, we can introduce

$$
u(\mathbf{x}):=\kappa(p(\mathbf{x})):=\int_{0}^{p(\mathbf{x})} k_{r}(\theta(s)) \mathrm{d} s
$$

as the Kirchhoff transformation of $p$. Here we assume $k_{r} \circ \theta$ to be Lipschitz and bounded with

$$
0<c_{l} \leq k_{r}(\theta(\cdot)) \leq c_{u}<\infty .
$$

Therefore we obtain

$$
\nabla u=\kappa^{\prime}(p) \nabla p=k_{r}(\theta(p)) \nabla p
$$

and problem (1) can be transformed to the following linear problem to find $u$ such that

$$
-\Delta u=f \quad \text { in } \Omega, \quad u=0 \quad \text { on } \Gamma_{D}, \quad \nabla u \cdot \mathbf{n}=g_{N} \quad \text { on } \Gamma_{N} .
$$




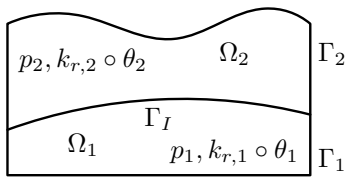

Figure 1: several domains

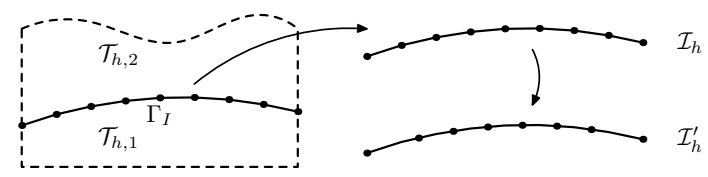

Figure 2: dual interface mesh

Obviously, such a simplification can not be achieved if $k_{r}$ depends in addition explicitly on $\mathbf{x} \in \Omega$. However, if this dependency is piecewise within non-overlapping subdomains $\Omega_{i}$, we can introduce local Kirchhoff transformations $u_{i}(\mathbf{x}):=\kappa_{i}\left(p_{i}(\mathbf{x})\right)$ for $\mathbf{x} \in \Omega_{i}$. So we can reformulate problem (1) and obtain the following nonlinear transmission problem, in the case of two subdomains, see Figure 1 , to find $u_{i}, i=1,2$, such that

$$
\left.\begin{array}{rlrl}
-\Delta u_{i} & =f & & \text { in } \Omega_{i}, \\
u_{i} & =0 & & \text { on } \Gamma_{D, i}, \\
\nabla u_{i} \cdot \mathbf{n}_{i} & =g_{N . i} & & \text { on } \Gamma_{N, i}, \\
\nabla u_{1} \cdot \mathbf{n}=\nabla u_{2} \cdot \mathbf{n}, \kappa_{1}^{-1}\left(u_{1}\right) & =\kappa_{2}^{-1}\left(u_{2}\right) & & \text { on } \Gamma_{I},
\end{array}\right\}
$$

where $\Gamma_{I}=\partial \Omega_{1} \cap \partial \Omega_{2}, \Gamma_{D, i}=\partial \Omega_{i} \cap \Gamma_{D}, \Gamma_{N, i}=\partial \Omega_{i} \cap \Gamma_{N}$ and $g_{N, i}=g_{N \mid \Gamma_{N, i}}$. The second, nonlinear interface condition ensures the continuity of the solution $p$ of the original problem (1), where we started from. Next we will derive a variational formulation for the nonlinear transmission problem (2).

\section{Primal-hybrid formulation}

In [2,3], an equivalent primal-hybrid formulation for the Poisson equation is presented. The idea is to introduce Lagrange multipliers acting on interfaces which ensure global continuity of the solution. We use this approach for the nonlinear transmission problem (2) and obtain the following variational problem to find $(u, \lambda) \in X \times M$, such that

$$
\begin{gathered}
\sum_{i=1}^{2} \int_{\Omega_{i}} \nabla u_{i} \cdot \nabla v_{i} \mathrm{~d} x+\int_{\Gamma_{I}} \lambda[v]_{\Gamma_{I}} \mathrm{~d} s_{x} \\
=\sum_{i=1}^{2} \int_{\Omega_{i}} f_{i} v_{i} \mathrm{~d} x+\sum_{i=1}^{2} \int_{\Gamma_{N, i}} g_{N, i} v_{i} \mathrm{~d} s_{x} \quad \text { for all } v \in X \\
\int_{\Gamma_{I}} \mu(u)_{\Gamma_{I}} \mathrm{~d} s_{x}=0 \quad \text { for all } \mu \in M
\end{gathered}
$$

with the ansatz spaces

$$
X:=\left\{v \in L_{2}(\Omega) \mid v_{i}=v_{\left.\right|_{\Omega_{i}}} \in H_{0, \Gamma_{D, i}}^{1}\left(\Omega_{i}\right), i=1,2\right\}, \quad M:=H_{00}^{\frac{1}{2}}\left(\Gamma_{I}\right)^{\prime},
$$

and with the jump terms

$$
[v]_{\Gamma_{I}}:=\left(v_{1}-v_{2}\right)_{\Gamma_{\Gamma_{I}}} \in M^{\prime} \quad \text { and } \quad(u)_{\Gamma_{I}}:=\left(\kappa_{1}^{-1}\left(u_{1}\right)-\kappa_{2}^{-1}\left(u_{2}\right)\right)_{\Gamma_{\Gamma_{I}}} \in M^{\prime} .
$$

*Corresponding author, e-mail gsell@tugraz.at 


\section{Discretization}

For the discretization of (3) we introduce the finite dimensional subspaces

$$
X_{h}:=\left\{v \in L_{2}(\Omega) \mid v_{i} \in S_{h}^{1}\left(\mathcal{T}_{h, i}\right), i=1,2\right\} \subset X
$$

and

$$
M_{h}:=\left\{\mu \in L_{2}\left(\Gamma_{I}\right) \mid \mu \in S_{h}^{0}\left(\mathcal{I}_{h}^{\prime}\right)\right\} \subset M,
$$

where $\mathcal{T}_{h, i}$ is an admissible triangulation of $\Omega_{i}$, and $\mathcal{I}_{h}^{\prime}$ is a modified dual mesh along the interface $\Gamma_{I}$ as depicted in Figure 2, see also [4]. For the solution of the nonlinear discrete problem we apply Newton's method by solving a sequence of the following linear problems. For given data $w_{h} \in X_{h}$, find $\left(u_{h}, \lambda_{h}\right) \in X_{h} \times M_{h}$, such that

$$
\left.\begin{array}{l}
\sum_{i=1}^{2} \int_{\Omega_{i}} \nabla u_{h, i} \cdot \nabla v_{h, i} \mathrm{~d} x+\int_{\Gamma_{I}} \lambda_{h}\left[v_{h}\right]_{\Gamma_{I}} \mathrm{~d} s_{x} \\
\left.=\sum_{i=1}^{2} \int_{\Omega_{i}} f_{i} v_{h, i} \mathrm{~d} x+\sum_{i=1}^{2} \int_{\Gamma_{N, i}} g_{N, i} v_{h, i} \mathrm{~d} s_{x} \quad \forall v_{h} \in X_{h}, \quad\right\} \\
\int_{\Gamma_{I}} \mu_{h}\left(w_{h}, u_{h}\right)_{\Gamma_{I}}^{\prime} \mathrm{d} s_{x} \\
\quad=\int_{\Gamma_{I}} \mu_{h}\left(w_{h}, w_{h}\right)_{\Gamma_{I}}^{\prime} \mathrm{d} s_{x}-\int_{\Gamma_{I}} \mu_{h}\left(w_{h}\right)_{\Gamma_{I}} \mathrm{~d} s_{x} \quad \forall \mu \in M_{h}
\end{array}\right\}
$$

where

$$
\left(w_{h}, u_{h}\right)_{\Gamma_{I}}^{\prime}:=\left(\left(\kappa_{1}^{-1}\right)^{\prime}\left(w_{h, 1}\right) u_{h, 1}-\left(\kappa_{2}^{-1}\right)^{\prime}\left(w_{h, 2}\right) u_{h, 2}\right)_{\left.\right|_{\Gamma_{I}}}
$$

is the linearization of the nonlinear jump $\left(u_{h}\right)_{\Gamma_{I}}$ and $w_{h}$ corresponds to the previous Newton iteration.

\section{Numerical example}

Consider $\mathbb{R}^{2} \supset \bar{\Omega}=[0,1]^{2}=([0,0.5] \times[0,1]) \cup([0.5,1] \times[0,1])=: \bar{\Omega}_{1} \cup \bar{\Omega}_{2}$, where $\Gamma_{N}=\{0\} \times(0,1)$ and $\Gamma_{D}=\partial \Omega \backslash \Gamma_{N}$. The local diffusion coefficients are chosen as in [1, Chapter 1]. The remaining data $f, g_{N, i}, g_{D, i}$ are given appropriately.
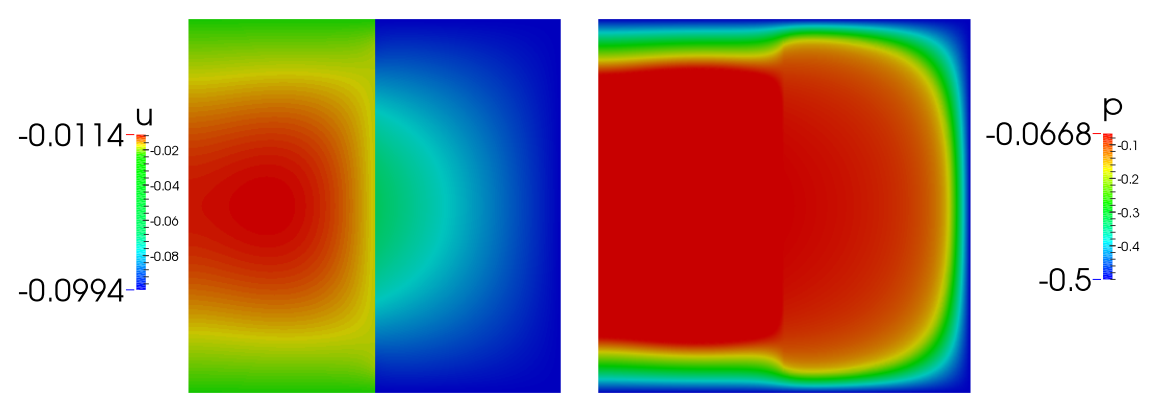

Figure 3: (discontinuous) solution $u$ of problem (4) and its (continuous) inverse Kirchhoff transformations $p_{i}=\kappa_{i}^{-1}\left(u_{i}\right), i=1,2$.

Acknowledgement: This work was supported by the Austrian Science Fund (FWF) within the IGDK 1754. 


\section{References}

[1] H. Berninger, Domain Decomposition Methods for Elliptic Problems with Jumping Nonlinearities and Application to the Richards Equation, $\mathrm{PhD}$ thesis, Freie Universität Berlin, 2008.

[2] D. Boffi, F. BREzZI, and M. Fortin, Mixed finite element methods and applications, Springer, Heidelberg, 2013.

[3] P. A. Raviart and J. M. Thomas, Primal hybrid finite element methods for 2nd order elliptic equations, Math. Comp. 31(138), 391-413 (1977).

[4] B. I. Wohlmuth, Discretization methods and iterative solvers based on domain decomposition, Springer, 2001. 